\title{
ESPECIE NUEVA DE WEINMANNIA (CUNONIACEAE) DE LOS BOSQUES ANDINOS ALTIMONTANOS DE LA PAZ, BOLIVIA
}

\begin{abstract}
Alfredo F. Fuentes
Herbario Nacional de Bolivia, Instituto de Ecología, Carrera de Biología, Universidad Mayor de San Andrés, Correo Central Cajón Postal 10077, La Paz, Bolivia. Missouri Botanical Garden, 63166-0299, St. Louis, Missouri, U.S.A. alfrefuentes@gmail.com (autor corresponsal).
\end{abstract}

\begin{abstract}
Fuentes, A. F. 2021. A new species of Weinmannia (Cunoniaceae) from the high-montane andean forests of La Paz, Bolivia. Darwiniana, nueva serie 9(1): 139-146.

Weinmannia epicae is described and illustrated. It grows in Andean high-montane forests of the Yungas region in the department of La Paz, northwestern Bolivia, between 2900 and $3300 \mathrm{~m}$. It differs from other morphologically similar species as $W$. cundinamarcensis, W. haenkeana and $W$. pubescens by having the maximum number of leaflets pairs usually between 10 to 14 , medial leaflets of 1.3 to $2.3 \mathrm{~cm}$, with more or less rough surface and undersurfaces with hirsute to hirsute-villous indument, and mature capsules villous-pubescent of 5-7 $\times 1.8-2.7 \mathrm{~mm}$, conspicuously pedicellated. The differences with morphological similar species are pointed out, and information on their geographical distribution, ecology, phenology and the assessment of conservation status according to IUCN criteria are provided.
\end{abstract}

Keywords. Flora; IUCN; Montane; taxonomy; Yungas.

Resumen. Fuentes, A. F. 2021. Especie nueva de Weinmannia (Cunoniaceae) de los Bosques andinos altimontanos de La Paz, Bolivia. Darwiniana, nueva serie 9(1): 139-146.

Se describe e ilustra Weinmannia epicae, especie nueva restringida a los bosques andinos altimontanos de la región de los Yungas del departamento de La Paz, en el noroeste de Bolivia, que crece entre 2900 a $3300 \mathrm{~m}$ de altitud. Se diferencia de otras especies morfológicamente similares como W. cundinamarcensis, $W$. haenkeana y $W$. pubescens por tener el número máximo de pares de foliolos usualmente entre 10 a 14 , foliolos mediales de 1,3-2,3 cm, con superficie más o menos rugosa e indumento villoso por el envés, y cápsulas maduras villoso-pubescentes de 5-7 × 1,8-2,7 mm, conspicuamente pediceladas. Se puntualizan las diferencias con especies similares, se provee información sobre su distribución geográfica, ecología, fenología y se presenta la evaluación del grado de amenaza según criterios de IUCN.

Palabras claves. Flora; IUCN; Montano; taxonomía; Yungas.

\section{INTRODUCCIÓN}

El género Weinmannia L. (Cunoniaceae, Cunonieae) comprende alrededor de 160 especies de árboles y arbustos distribuidas en los trópicos y subtrópicos del mundo (Bradford et al., 2004; Fuentes \& Rogers, 2007; Morales, 2010, 2018). En América se han registrado 86 especies (Ulloa et al., 2017), concentradas en los Andes y 21 de ellas crecen en Bolivia (Harling \& Fuentes, 2014).
Las especies de Weinmannia son muy variables morfológicamente, lo cual dificulta su taxonomía. Esta variación es atribuida a posibles eventos de hibridación entre sus especies (Harling, 1999; Fuentes 2011; Morales 2010) cuando comparten sus áreas de distribución, particularmente en filos de serranías de los pisos altimontano y montano, aproximadamente entre 2500 a 3500 m s.m. Por otro lado, la amplia distribución y variación morfológica de algunas especies como 
Weinmannia pinnata L., que se distribuye desde el sur de Mexico y las Antillas Mayores hasta el centro de Bolivia (Morales, 2010), sugieren la existencia de posibles complejos de especies crípticas que probablemente requieran de datos moleculares para distinguirlas.

Exceptuando el tratamiento taxonómico de las especies de Weinmannia de Mexico y Centroamérica (Morales, 2010), el resto de las áreas neotropicales no cuentan todavía con estudios exhaustivos recientes de este género. Aunque tratamientos de Cunoniaceae en catálogos recientes como el de Bolivia (Fuentes \& Harling, 2014 ) y de las Américas (Ulloa et al., 2017) han permitido esclarecer algunas cuestiones nomenclaturales, las especies de Weinmannia de la región andina son las que requieren de mayores estudios, pues su taxonomía es confusa.

En cuanto al estado de conservación de las especies presentes en Bolivia, seis especies han sido evaluadas a nivel global, de las cuales cinco se encuentran en la categoría de preocupación menor LC (Weinmannia balbisiana Kunth, W. crassifolia Ruiz \& Pav., W. fagaroides Kunth, $W$. pinnata y $W$. trianaea Wedd.) y una como vulnerable $\mathrm{Vu}(W$.yungasensis A. Fuentes \& Z.S. Rogers) (IUCN, 2021). Por otro lado, en evaluaciones a nivel nacional tres especies han sido catalogadas como vulnerables VU (W. bangii Rusby, $W$. boliviensis R.E. Fr. y $W$. davidsonii A. Fuentes \& Z.S. Rogers) y una en peligro EN ( $W$. fagaroides) (Ministerio de Medio Ambiente y Agua, 2012).

En el presente trabajo se describe una especie nueva andina de Weinmannia como resultado del estudio de colecciones de la región del Madidi en el noroeste de Bolivia.

\section{MATERIALES Y MÉTODOS}

El material tipo y la mayor parte de los especímenes de esta especie nueva fueron coleccionados dentro de las actividades del Proyecto Madidi, que se lleva a cabo desde el 2001 en una de las regiones más biodiversas del mundo en el NO de Bolivia. Esta región cubre un gradiente altitudinal desde los 200 hasta los $6000 \mathrm{~m}$ s.m., en el que se encuentran ecosistemas andinos, amazónicos y de valles secos (Fuentes, 2005). Este proyecto es una colaboración entre el Herbario Nacional de Bolivia, el Jardín Botánico de Misuri y la Universidad Autónoma de Madrid.
Entre sus resultados más relevantes se incluyen el corroborar que el Parque Madidi es uno de los más ricos en especies de plantas del mundo (Fuentes, 2018). Además se registraron más de 200 especies nuevas de plantas vasculares, entre ellas dos de Weinmannia (Fuentes \& Rogers, 2007). Detalles de los resultados del Proyecto Madidi se pueden obtener en la página web https://madidiproject.weebly.com/

Especímenes adicionales de esta especie nueva y de otras similares fueron estudiadas en la colección del Herbario Nacional de Bolivia (LPB), que cuenta con un juego representativo de colecciones de Weinmannia sudamericanas. La descripción morfológica y fenológica, distribución geográfica, caracterización ecológica, y la evaluación del grado de conservación están basados en 16 especímenes.

Tambien se consultaron herbarios o repositorios de especímenes botánicos virtuales como JSTOR (https://plants.jstor.org/, consultas junio y julio del 2019), y del Field Museum (https://plantidtools. fieldmuseum.org/es/rrc/5581, consultas junio y julio del 2019) entre los principales.

Los acrónimos de los herbarios son citados según Thiers (2020). Para la terminología sobre el indumento se sigue a Hewson (1988). La evaluación del estado de conservación se la realizó siguiendo los lineamientos de la IUCN (IUCN, 2012; 2017).

\section{RESULTADOS Y DISCUSIÓN}

Weinmannia epicae A. Fuentes sp. nov. TIPO: Bolivia. Departamento La Paz, prov. Franz Tamayo, Parque Nacional Madidi, entre Chuncani e Ichocorpa, por el camino de herradura entre Keara y Mojos, bosque bajo yungueño altimontano pluvial de

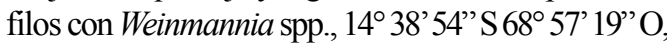
2900 m s.m., 27-IV-2016, A. Fuentes 20062 (Holótipo: LPB!; Isótipos: BOLV!, LPB!, MO!) (Figs. 1 y 2).

Weinmannia epicae differs from similar Andean species with compound leaves and conspicuous indument as $W$. cundinamarcensis, $W$. haenkeana and $W$. pubescens by the following combination of characters: maximum number of leaflets pairs 10-18 (vs. less than 14), more or less rough leaflets (vs. plane or concave-rugose), long pedicels up to $3 \mathrm{~mm}$ (vs. less than $1.2 \mathrm{~mm}$ ), long capsules up to $7 \mathrm{~mm}$ (vs. les than $5 \mathrm{~mm}$ ). 


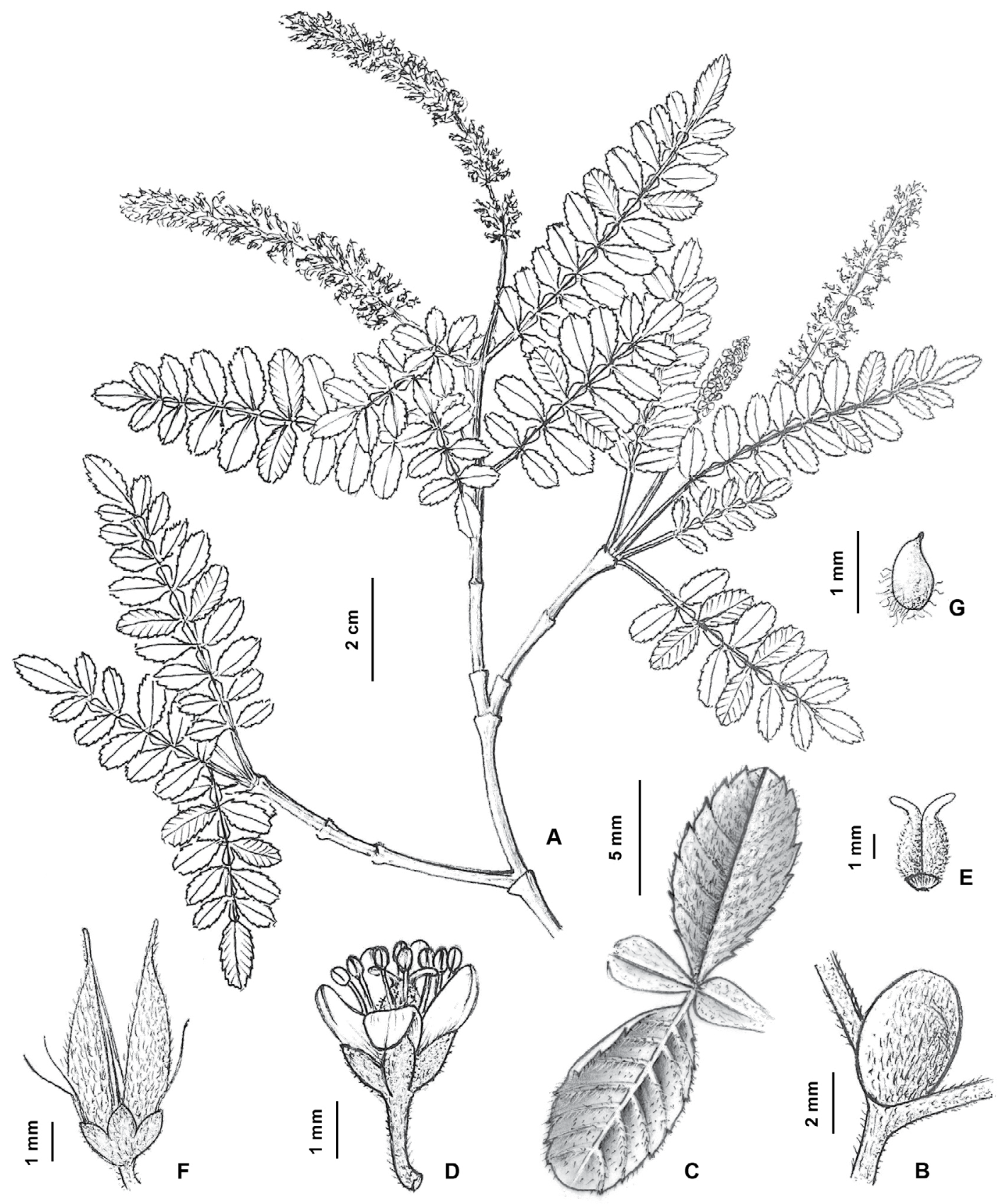

Fig. 1. Weinmannia epicae. A, rama fértil. B, estípula. C, detalle de los folíolos mediales vistos por el haz y envés. D, detalle de la flor. E, ovario. F, fruto maduro. G, semilla. A-E y G, holótipo Fuentes 20062 (LPB); F, Beck 17703 (LPB).

Arbolito, árbol a arbusto, altura reproductiva 2,5-10(16) $\mathrm{m}$ y hasta $27 \mathrm{~cm}$ de dap; entrenudos 0,9$4,6 \mathrm{~cm}$; ramas pardas a pardo-rojizas en vivo cuando jóvenes, planas dorsoventralmente cerca de los nudos cuando jóvenes, después subcuadrangulares o teretes, densamente hirsutas a hirsuto-villosas de jóvenes, los tricomas 0,5-1,5 mm. Estípulas caducas, anchamente ovadas, 4,5-9,5 × 4-9 mm, 

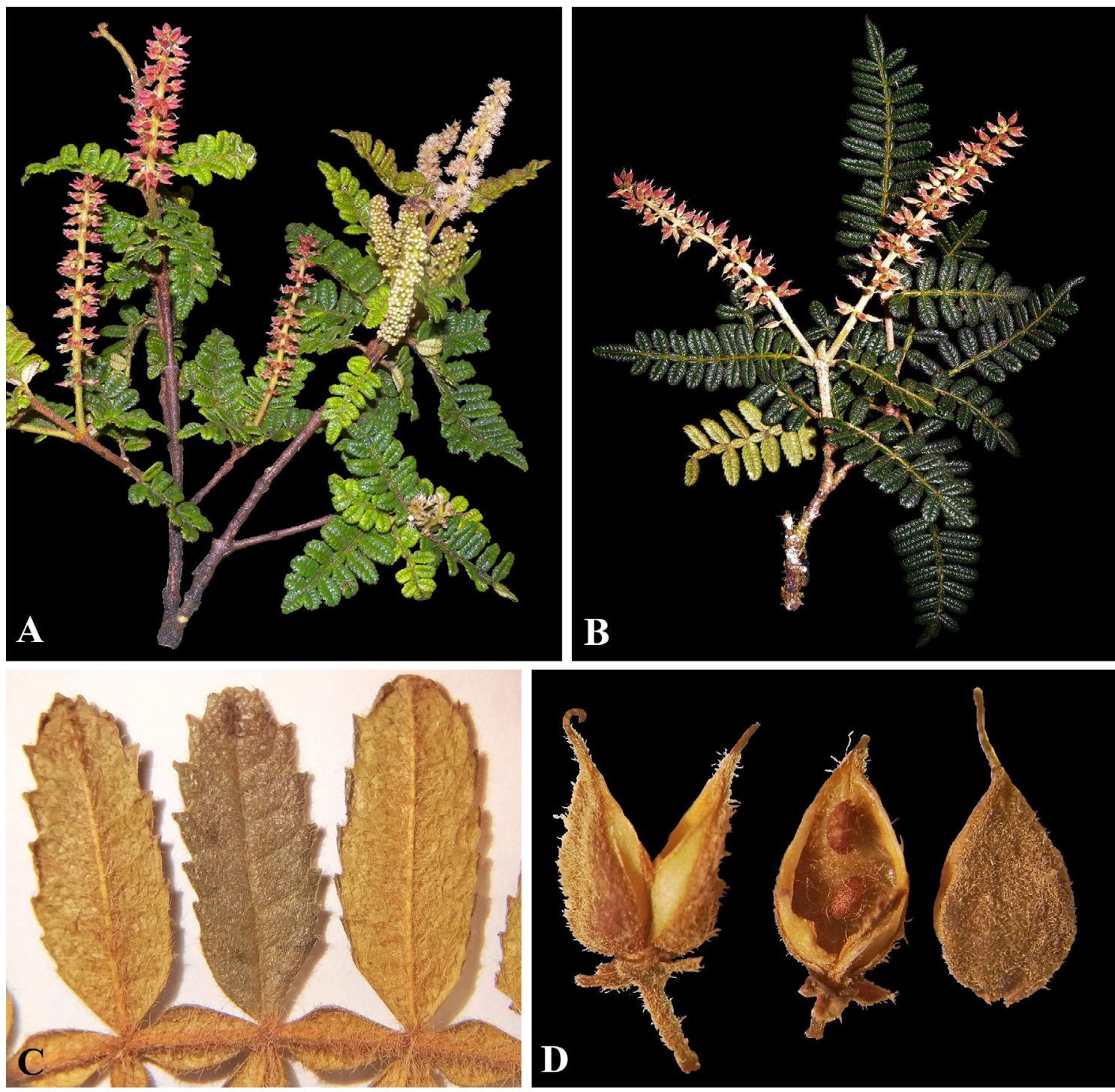

Fig 2. Weinmannia epicae. A, rama florífera. B, rama fructífera. C, detalle de los folíolos mediales. D, fruto maduro mostrando una valva en vista ventral con dos semillas y otra en vista dorsal. A, tipo Fuentes 20062 (LPB); B y C, Fuentes \& Ampuero 16879 (LPB); D, Beck 17703 (LPB). Figura en color en la versión en línea http://www.ojs.darwin. edu.ar/index.php/darwiniana/article/view/934/1214

subcartáceas, glabras por el haz, densamente blanco hirsutas a pubescentes por el envés, la base auriculada, el margen entero, el ápice redondeado. Hojas opuestas, decusadas, verde claras a pardo claras cuando secas, compuestas, con (4)5-14(18) pares de foliolos (número máximo 10-14(18) pares); pecíolo 4-9(12,5) $\mathrm{mm}$, villoso a hirsutovilloso, con tricomas de 0,7-2 $\mathrm{mm}$, levemente canalado a plano adaxialmente, triangularredondeado abaxialmente, sin alas; raquis $(1,5-) 3-$ $8(-11) \mathrm{cm}$, con alas revolutas e hirsuto-villosas, de 3,5-5,5 $\mathrm{mm}$ de ancho en la parte media; folíolos más o menos rugosos, a veces casi planos, cartáceos a coriáceos, laxamente hirsuto-villosos adaxialmente, villosos a hirsuto-villosos con tricomas más largos de hasta 1,8 $\mathrm{mm}$ abaxialmente, 
el margen en su mayor parte aserrado, entero hacia la base, revoluto, el nervio central plano a ligeramente prominente adaxialmente, claramente prominente abaxialmente, nervios de orden menor planos a ligeramente inmersos adaxialmente, levemente prominentes abaxialmente; folíolo terminal frecuentemente lanceolado, a veces elíptico u ovado, 1,1-2,3(-2,7) × 0,4-0,7(-0,9) cm, la base y el ápice frecuentemente agudos, el ápice a veces redondeado; folíolos laterales oblongos, a veces elípticos u ovados, los dos a tres pares basales algo más pequeños que el tercer o cuarto par, luego reducen gradualmente su tamaño hacia el ápice del raquis, $0,9-2,1(-2,4) \times(0,3-) 0,5-0,8(-1) \mathrm{cm}$, la base obtusa a aguda, asimétrica, el ápice redondeado a agudo.Inflorescencias(4,6-)6-8,5(-10,2)×0,9-1,2cm, axilares, pseudoracemosas, en pares hacia el ápice de las ramas, laxas; pedúnculos (0,9-)1,3-1,8(-2,5)× ca. $0,1 \mathrm{~cm}$, tetragonales, hirsuto-villosos; raquis 5-7,5 cm, hirsuto-villosos; brácteas florales oblongolineares, a veces ovadas, 1-1,5 ×0,4-0,7 mm, glabras adaxialmente, abaxialmente hirsutas, subtendiendo fascículos de (2)3 o 4 flores. Flores bisexuales, 4- raro 5-meras, blancas a blancas con matices rosados en fresco; pedicelo 1,2-3 × 0,2-0,3 mm, estrigoso-hirsuto; sépalos 4 o 5 , triangulares, 1,1$1,3 \times 1-1,1 \mathrm{~mm}$, cartáceos, hirsuto villosos, el margen entero, el ápice agudo, ciliado; pétalos 4 o 5 , oblongos a elípticos, 1-1,4 × 0,8-1,1 mm, membranáceos, con algunos tricomas hacia el nervio central por el envés, el margen entero, el ápice obtuso o redondeado, el nervio central evidente; estambres 8 o 10, los filamentos 2,5-3 mm, glabros, las anteras reniformes, ca. $0,5 \mathrm{~mm}$; nectario floral cupuliforme, ca. 0,4-0,6 mm, 1,2$1,4 \mathrm{~mm}$ diam., con 8 o 10 surcos en los cuales se apoyan las bases de los filamentos; ovario bilocular, ovoide, 1,5-1,8 × 0,7-1 mm (longitud incluyendo los estilos), blanquecino-estramíneo en seco, hirsuto; estilos 2, 1-1,5 mm, glabros, a veces rojo-violáceos, estigma clavado. Fruto cápsula septicida, 2-valvada, ovada o elíptica, 5-7 × 1,82,7 $\mathrm{mm}$ (longitud incluyendo los estilos), rojiza de inmadura, pardo oscura de madura, villosapubescente, con una o varias líneas longitudinales en la superficie externa; estilos persistentes 2-3 mm; semillas reniformes, 0,9-1,1 $\times 0,5 \mathrm{~mm}$, pardas, laxamente villosas sobre el dorso, los tricomas pardo-amarillentos, 0,9-1,5 $\mathrm{mm}$.
Nombres vulgares. "Monte calvario", "kepu wichullo", "wichullo" (informantes locales de la región del Madidi).

Etimología. El epíteto rinde homenaje a mi madre Epifania Claros, cariñosamente llamada Epica.

Distribución geográfica y ecología. Conocida solamente de los bosques de la ceja de monte inferior, en áreas con climas pluviales, del departamento de La Paz, Bolivia, entre 2900 a $3300 \mathrm{~m}$ s.m., en las cordilleras de Apolobamba y La Paz (Fig. 3). Biogeográficamente ocupan territorios del sector "Yungas de la cuenca alta del Beni" (Navarro, 2011) en la provincia biogeográfica de los Yungas (Rivas-Martínez et al., 2011). Esta especie crece en bosques primarios y clareados, junto con especies leñosas como Brunellia boliviana Britton ex Rusby var. brittonii (Rusby) Cuatrec. (Brunelliaceae); Chusquea spp. (Poaceae); Clethra scabra Pers. var. laevigata (Meisn.) Sleumer (Clethraceae); Clusia multiflora Kunth; C. sphaerocarpa Planch. \& Triana (Clusiaceae); Cyathea austropallescens Lehnert, C. parvifolia Sodiro (Cyatheaceae); Desfontainia spinosa Ruiz \& Pav. (Columelliaceae); Freziera apolobambensis D. Santam. \& A. Fuentes (Pentaphylacaceae); Hedyosmum dombeyanum Solms, H. racemosum (Ruiz \& Pav.) G. Don (Chloranthaceae); Ilex pseudoebenacea Loes. (Aquifoliaceae); Miconia quadrialata S.S. Renner \& S. Beck, M. setulosa Cogn. (Melastomataceae); Myrsine coriacea (Sw.) R. Br. ex Roem. \& Schult., M. dependens (Ruiz\& Pav.) Spreng. (Primulaceae); Symplocos polyphylla B. Ståhl (Symplocaceae); Weinmannia davidsonii, W. reticulata Ruiz \& Pav. y W. yungasensis (Cunoniaceae).

Fenología. Weinmannia epicae ha sido coleccionada con flores en abril y con frutos entre abril y agosto.

Usos. Se usa localmente como leña.

Estado de conservación. El área de distribución de Weinmannia epicae se restringe al departamento de La Paz en el noroeste de Bolivia, en el piso altimontano o ceja de monte entre 2900 a $3300 \mathrm{~m}$ s.m. de las cordilleras de Apolobamba y 
La Paz, un rango altitudinal con fuertes presiones actuales y futuras, pues es de estos bosques que los pobladores locales extraen leña, y además queman los bosques y pajonales como parte de sus actividades agrícolas, pero sobre todo para el pastoreo de ganado. Además, en los últimos años con la intensificación de la actividad minera en estas áreas, las presiones han aumentado. Weinmannia epicae tiene una EOO de $1663 \mathrm{~km}^{2}$ y un AOO de $32 \mathrm{~km}^{2}$. Por las anteriores razones la especie es provisionalmente categorizada como En Peligro (ENB1ab+B2ab).

Discusión. Weinmannia epicae pertenece a un grupo morfológico de especies andinas caracterizado por tener hojas compuestas, con indumento conspícuo y ovario pubescente a tomentoso como $W$. cundinamarcensis Cuatrec., $W$. haenkeana Engl. y $W$. pubescens Kunth que corresponde a la serie "Pubescentes" de la clasificación informal de Bernardi (1961), las dos primeras presentes en Bolivia. Weinmannia epicae difiere de las especies anteriores fundamentalmente por tener mayor número de pares de folíolos y frutos más grandes (Tabla 1). Weinmannia epicae se asemeja más a $W$. haenkeana, por presentar hojas rugosas y tener elevado número máximo de pares de folíolos por hoja, sin embargo difiere por su inflorescencia más o menos laxa dejando ver el raquis vs. muy congesta no dejando ver el raquis, y por el indumento del envés de los folíolos que se distribuye en toda la superficie vs. concentrado hacia el nervio central. Difiere de $W$. cundinamarcensis por tener mayor número de pares de folíolos (18 vs. 10), folíolos rugosos (vs. lisos), pedicelos fructíferos más largos (1,23 vs. $0,3-0,7 \mathrm{~mm})$ y frutos más grandes $(5-7 \mathrm{x}$ 1,8-2,7 vs. $2,2-3 \times 1-1,2 \mathrm{~mm})$. De $W$. pubescens (que no se encuentra en Bolivia), difiere además de los anteriores caracteres, por el indumento de tricomas más largos de hasta $1,8 \mathrm{~mm}$ (vs. de menos de $1 \mathrm{~mm}$ ) en el envés de las hojas. Weinmannia lechleriana Engl. es otra especie de este grupo con la que sobrepone su distribución geográfica, aunque se encuentra a menores elevaciones; difiere principalmente por el indumento villoso a hirsutovilloso de las hojas (vs. piloso a glabrescente). Las principales diferencias con especies afines de este grupo se encuentran sintetizadas en la tabla 1.

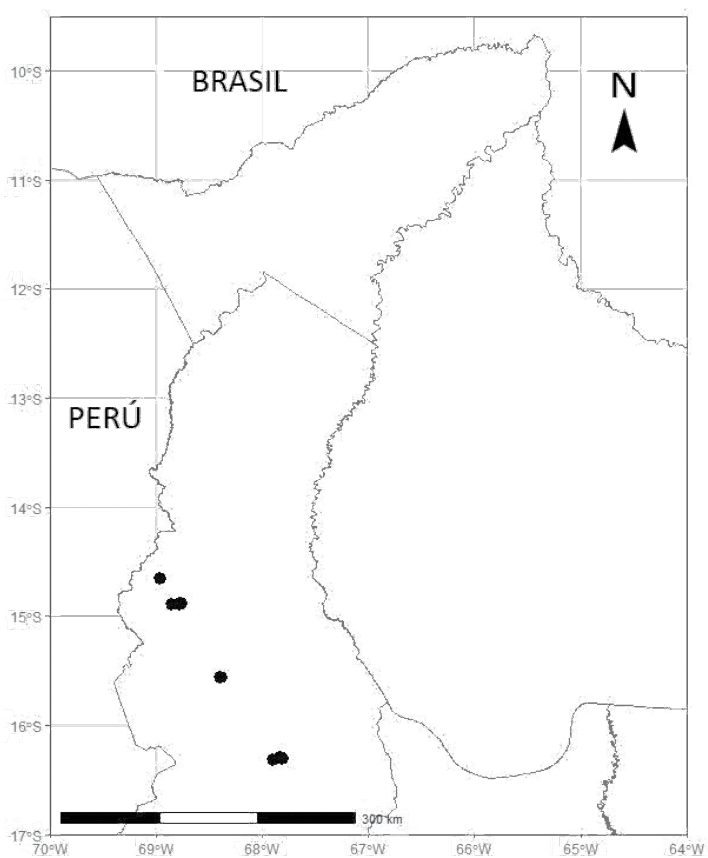

Fig 3. Mapa de distribución de Weinmannia epicae.

\section{Material representativo examinado}

BOLIVIA. La Paz. Prov. Bautista Saavedra, Parque Nac. Madidi, comunidad Laji Sorapata, sector Supay Puncu, 2917 m s.m., 14 53' 12" S 68 50' 59" O, 27-VI-2010, A. Fuentes \& $M$. Ampuero 16879 (LPB, MO); Área Natural de Manejo Integrado Apolobamba, Hilo Hilo, sector Chaka, 3189 m s.m., $14^{\circ} 53^{\prime} 00^{\prime \prime} \mathrm{S} 68^{\circ} 46^{\prime}$ 53" O, 18-VIII-2009, L. Cayola, M. Reguerin, O. Paredes, E. Chavez \& J. Corhuari 3451 (LPB, MO). Prov. Franz Tamayo, Parque Nac. Madidi, sector Quimsa Cruz, entre Calla y Tokoake, 3300 m s.m., $14^{\circ} 39^{\prime} 18^{\prime \prime} \mathrm{S} 68^{\circ}$ 57' 47" W, 25-VI2005, A. Fuentes, I. Jiménez-Pérez, R. Hurtado \& R. Cuevas 8718 (BOLV, CTES, GB, LPB, MO, USZ). Prov. Larecaja, Cocapunco, bajando por el camino antiguo Ingenio-Mapiri, $2888 \mathrm{~m}$ s.m., $15^{\circ} 33$ ' 22" S 68 23' 43" O, 9-10-VI-2010, G. Arellano, P. Soliz, O. Quiroga, J. Salas, B. Apaza \& M. Chacón 2788 (LPB, MA, MO). Prov. Nor Yungas, de la cumbre bajando $30 \mathrm{~km}$ hacia Chuspipata, $3150 \mathrm{~m}$ s.m., $16^{\circ} 17^{\prime} 21^{\prime \prime} \mathrm{S} 67^{\circ} 49^{\prime}$ 38" O, 21-VI-1990, S. Beck 17703 (BOLV, CTES, GB, K, LPB, M, MO, NY, USZ). 
Tabla 1. Principales diferencias entre Weinmannia epicae A. Fuentes y las especies morfológicamente relacionadas.

\begin{tabular}{|c|c|c|c|c|c|}
\hline Caracter & $\begin{array}{l}\text { Weinmannia } \\
\text { epicae }\end{array}$ & $\begin{array}{l}\text { Weinmannia } \\
\text { haenkeana }\end{array}$ & $\begin{array}{l}\text { Weinmannia } \\
\text { cundinamarcensis }\end{array}$ & $\begin{array}{l}\text { Weinmannia } \\
\text { pubescens }\end{array}$ & $\begin{array}{l}\text { Weinmannia } \\
\text { lechleriana }\end{array}$ \\
\hline $\begin{array}{l}\text { Pares de foliolos por } \\
\text { hoja }\end{array}$ & $(3-) 5-14(18)$ & $4-8(13)$ & $(4) 5-9(10)$ & $4-6(-8)$ & $(2) 3-7(-10)$ \\
\hline $\begin{array}{l}\text { Núm. máximo de pares } \\
\text { de foliolos }\end{array}$ & $10-14(18)$ & $7-10(13)$ & $8-9(10)$ & $5-8$ & $5-7(10)$ \\
\hline Superficie del envés & rugosa & concavo-rugosa & lisa & lisa & lisa \\
\hline Pubescencia del envés & hirsuto-villosa & $\begin{array}{l}\text { villosa, } \\
\text { concentrada en } \\
\text { nervio central }\end{array}$ & pubescente & pubescente & $\begin{array}{l}\text { pilosa a } \\
\text { glabrescente }\end{array}$ \\
\hline Inflorescencia & $\begin{array}{l}\text { fasciculos } \\
\text { separados }\end{array}$ & congesta cilíndrica & $\begin{array}{l}\text { fasciculos } \\
\text { separados }\end{array}$ & $\begin{array}{l}\text { fasciculos } \\
\text { separados }\end{array}$ & $\begin{array}{l}\text { fasciculos } \\
\text { separados }\end{array}$ \\
\hline $\begin{array}{l}\text { Long. pedicelo } \\
\text { fructífero }(\mathrm{mm})\end{array}$ & $1,2-3$ & $0,2-0,8$ & $0,3-0,7$ & $0,2-1,2$ & $0,9-3(-5)$ \\
\hline Tamaño del fruto (mm) & $5-7 \times 1,8-2,7$ & $4,5-5 \times 1,1-1,9$ & $2,2-3 \times 1-1,2$ & $2-3,5 \times 1,1-1,5$ & $3-4 \times 1-1,3$ \\
\hline Rango elev. (m s.m.) & $2800-3300$ & $2200-3300$ & $1900-3100$ & $1500-3400$ & $1300-2600$ \\
\hline Distribución & Bolivia & Perú y Bolivia & $\begin{array}{l}\text { Colombia, Perú, } \\
\text { Bolivia }\end{array}$ & $\begin{array}{l}\text { Venezuela, } \\
\text { Colombia, Perú, } \\
\text { Ecuador }\end{array}$ & $\begin{array}{l}\text { Venezuela, } \\
\text { Colombia, Perú, } \\
\text { Bolivia }\end{array}$ \\
\hline
\end{tabular}

\section{AGRADECIMIENTOS}

A Don Carlos Maldonado por elaborar la ilustración. El trabajo de campo se llevó a cabo gracias al soporte de fondos del Missouri Botanical Garden MBG-CFD0036B, National Science Foundation NSF0743457, National Geographic NGS 03052 y de la familia Davidson DAV 0237. A los herbarios Nacional de Bolivia, de la Universidad Nacional Mayor de San Marcos y del Missouri Botanical Garden. A los revisores anónimos y al editor por sus contribuciones en la mejora del presente trabajo.

\section{BIBLIOGRAFÍA}

Bernardi, L. 1961. Revisio generis Weinmanniae. Pars I: Sectio Weinmanniae. Candollea 17: 123-189.

Bradford, J. C.; H. C. Fortune-Hopkins \& R. W. Barnes. 2004. Cunoniaceae, in K. Kubitzki (ed.), The Families and Genera of Vascular Plants, 6: 91-111. Springer Verlag, Berlin. DOI: https://doi.org/10.1007/978-3-66207257-8_11

Fuentes, A. F. 2005 [2006]. Una introducción a la vegetación de la región del Madidi. Ecología en Bolivia 40: 1-31.

Fuentes, A. F. 2011. Cunoniaceae. En A. Carretero; M. Serrano, F. Borchsenius \& H. Balslev (eds.). Pueblos y Plantas de Chuquisaca: Estado del Conocimiento de los pueblos, la flora, uso y conservación, pp.133-135. Herbario del Sur de Bolivia-Universidad Mayor, Real y Pontificia de San Francisco Xavier de Chuquisaca, Sucre. Fuentes, A. F. 2018. Novedades florísticas de la región del Madidi: nuevos registros de plantas vasculares, adiciones al catálogo de Bolivia y especies poco conocidas. Kempffiana 14: 42-62.

Fuentes, A. F. \& Z. S. Rogers. 2007. Dos especies nuevas de Weinmannia (Cunoniaceae) de los bosques montanos en La Paz, Bolivia. Novon 17: 326-331. DOI: http://dx.doi. org/10.3417/1055-3177(2007)17\%5B326:DENDWC\%5D 2.0.CO;2

Harling, G. 1999. Cunoniaceae, in G. Harling \& L. Andersson (eds.), Flora of Ecuador, 61: 1-74. Council of Nordic Publications in Botany, Copenhagen.

Harling, G. \& A. F. Fuentes. 2014. Cunoniaceae, in P. M. Jørgensen, M. H. Nee \& S. G. Beck (eds.), Catálogo de las Plantas Vasculares de Bolivia. Monographs in Systematic Botany from the Missouri Botanical Garden 127: 540-542.

Hewson, H. J. 1988. Plant Indumentum - a handbook of terminology. Australian Flora and Fauna Series 9. Australian Government Publishing Service, Canberra.

IUCN. 2012. Categorías y criterios de la lista roja de la Unión International para la Conservación de la Naturaleza UICN. Versión 3.1. Gland, Suiza. Segunda 
edición. https://www.iucn.org/es/content/categorias-ycriterios-de-la-lista-rojade-la-uicn-version-31-segundaedicion

IUCN. 2017. Standards and Petitions Subcommitee. Guidelines for Using the IUCN Red List Categories and Criteria. Version 13. Prepared by the Standardsand Petitions Subcommitee. http://www.iucnredlist.org/ documents/RedListGuidelines.pdf

IUCN. 2021. The IUCN Red List of Threatened Species. Version 2021-1. https://www.iucnredlist.org, Consultado [1 Abril 2021].

Ministerio de Medio Ambiente y Agua. 2012. Libro Rojo de la Flora amenazada de Bolivia. Vol. I. Zona Andina. Ministerio de Medio Ambiente y Agua, La Paz.

Morales, J. F. 2010. Sinopsis del género Weinmannia (Cunoniaceae) en México y Centroamérica. Anales del Jardín Botánico de Madrid 67(2): 137-155.

Morales, J. F. 2018. New species and combinations of Apocynaceae, Bignoniaceae, Clethraceae, and Cunoniaceae from the Neotropics. Anales del Jardin Botánico de Madrid 75(2): 1-10.
Navarro, G. 2011. Clasificación de la vegetación de Bolivia. Editorial Centro de Ecología Difusión Simón I. Patiño, Santa Cruz, Bolivia.

Rivas-Martínez S.; G. Navarro, A. Penas \& M. Costa. 2011. Biogeographic Map of South America. A preliminary survey. International Journal of Geobotanical Research 1: 21-40 + Map.

Thiers, B. 2020. [continuamente actualizado] Index Herbariorum: A global directory of public herbaria and associated staff. New York Botanical Garden's Virtual Herbarium. Available from http://sweetgum.nybg.org/ science/ih/

Ulloa Ulloa, C.; P. Acevedo-Rodríguez, S. G. Beck, M. J. Belgrano, R. Bernal, P. E. Berry, L. Brako, M. Celis, G. Davidse, S. R. Gradstein, O. Hokche, B. León, S. LeónYánez, R. E. Magill, D. A. Neill, M. H. Nee, P. H. Raven, H. Stimmel, M. T. Strong, J. L. Villaseñor Ríos, J. L. Zarucchi, F. O. Zuloaga \& P. M. Jørgensen. 2017. An integrated assessment of vascular plants species of the Americas. Science 358: 1614-1617. DOI: http://dx.doi. org/10.1126/science.aao0398 